\title{
Visualization of basement membranes in normal breast and breast cancer tissues using multiphoton microscopy
}

\author{
XIUFENG WU ${ }^{1,2^{*}}$, GANG CHEN $^{3 *}$, JINGTING QIU ${ }^{4}$, JIANPING LU ${ }^{3}$, WEIFENG ZHU ${ }^{3}$, \\ JIANXIN CHEN ${ }^{4}$, SHUANGMU ZHUO ${ }^{4}$ and JUN YAN ${ }^{1,2}$ \\ ${ }^{1}$ Department of Surgery, Fujian Provincial Tumor Hospital, Teaching Hospital of Fujian Medical University, Fuzhou, \\ Fujian 350014; ${ }^{2}$ Department of General Surgery, Nanfang Hospital, Southern Medical University, Guangzhou, \\ Guangdong 510515; ${ }^{3}$ Department of Pathology, Fujian Provincial Tumor Hospital, Teaching Hospital of Fujian \\ Medical University, Fuzhou, Fujian 350014; ${ }^{4}$ Institute of Laser and Optoelectronics Technology, Fujian Provincial \\ Key Laboratory for Photonics Technology, Key Laboratory of Optoelectronic Science and Technology for \\ Medicine of the Ministry of Education, Fujian Normal University, Fuzhou, Fujian 350007, P.R. China
}

Received January 20, 2015; Accepted March 18, 2016

DOI: $10.3892 / \mathrm{ol} .2016 .4472$

\begin{abstract}
Since basement membranes represent a critical barrier during breast cancer progression, timely imaging of these signposts is essential for early diagnosis of breast cancer. A label-free method using multiphoton microscopy (MPM) based on two-photon excited fluorescence signals and second harmonic generation signals for analyzing the morphology of basement membrane in normal and cancerous breast tissues is likely to enable a better understanding of the pathophysiology of breast cancer and facilitate improved clinical management and treatment of this disease. The aim of this study was to determine whether MPM has the potential for label-free assessment of the morphology of basement membrane in normal and cancerous breast tissues. A total of 60 tissue section samples (comprising 30 fresh breast cancer specimens and 30 normal breast tissues) were first imaged (fresh, unfixed and unstained) with MPM and are then processed for routine hematoxylin and eosin (H\&E) histopathology. Comparisons were made between MPM imaging and gold standard sections for each specimen stained with H\&E. Simply by visualizing morphological
\end{abstract}

Correspondence to: Dr Jun Yan, Department of General Surgery, Nanfang Hospital, Southern Medical University, 1838 North Guangzhou Avenue, Guangzhou, Guangdong 510515, P.R. China

E-mail: ynjun@yahoo.com

Mr. Shuangmu Zhuo, Institute of Laser and Optoelectronics Technology, Fujian Provincial Key Laboratory for Photonics Technology, Key Laboratory of Optoelectronic Science and Technology for Medicine of the Ministry of Education, Fujian Normal University, 8 Shangshan Road, Fuzhou, Fujian 350007, P.R. China

E-mail: shuangmuzhuo@gmail.com

*Contributed equally

Key words: multiphoton microscopy, basement membrane, breast cancer features appearing on multiphoton images, cancerous lesions may be readily identified by the loss of basement membrane and tumor cells characterized by irregular size and shape, enlarged nuclei and increased nuclear-cytoplasmic ratio. These results suggest that MPM has potential as a label-free method of imaging the morphology of basement membranes and cell features to effectively distinguish between normal and cancerous breast tissues.

\section{Introduction}

Breast cancer is the most frequently diagnosed cancer and the leading cause of cancer-related mortality among females, accounting for $23 \%$ of all cancer cases and $14 \%$ of cancer-related mortalities (1). Metastasis involves a multistep process including the detachment of cancer cells from a primary site, the invasion of surrounding tissue, and spreading through the circulation, and represents one of the main causes of mortality in breast cancer patients. In recent years, disease-related mortality and metastasis have declined as a result of adjuvant therapy. Comprehensive therapy (including chemotherapy and endocrine therapy) facilitates the suppression of the metastatic dissemination of local tumors (2). However, these treatments target the tumor cells and disregard the basement membrane, which represents a critical barrier during breast cancer progression. With breast cancer progression, tumor cells invade surrounding tissues and spread to distant organs, eventually leading to metastasis by inducing disruption of the underlying basement membrane (3), as shown in Fig. 1. Basement membranes play a key role in tumor progression (4). However, these signposts of tumor progression have so far only been evaluated by histological examination of previously excised specimens $(5,6)$. It would therefore be useful to have a tool that allows label-free in situ imaging of these signposts.

The basement membrane is located between the epithelium and stroma. Since epithelial cells are able to generate a two-photon excited fluorescence (TPF) signal and the stroma 
is composed primarily of collagen that is capable of emitting a strong second harmonic generation (SHG) signal (7-10), multiphoton microscopy (MPM) based on TPF and SHG signals may be useful for visualizing the outline of basement membranes that are not detectable by other imaging modalities.

As a nonlinear optical technique, MPM has advantages including label-free imaging, inherent optically sectioning, deep optical penetration, and reduced specimen photobleaching and photodamage (11-15). In this study, we used MPM to visualize the in situ morphology of basement membrane in normal and cancerous breast tissues based on intrinsic nonlinear optical contrast. Our results reveal that MPM demonstrates marked differences in the organization of basement membranes in normal breast tissue and breast cancer tissue.

\section{Materials and methods}

Tissue specimens. A total of 30 fresh breast biopsy specimens were obtained from 30 patients who underwent biopsy and were later diagnosed with breast cancer by conventional histological modality. Thirty normal breast tissues were obtained from reduction surgeries performed at Fujian Provincial Tumor Hospital, China. Written informed consent was required from every participant according to a protocol approved by the Institutional Review Board of Fujian Provincial Tumor Hospital and in accordance with the Declaration of Helsinki. The specimens were placed in a glass-bottomed dish (coverglass, 0.085-0.13 mm; MatTek Corporation, Ashland, MA, USA) for multiphoton imaging. In this study, the specimen preparation and multiphoton imaging were completed within $1 \mathrm{~h}$ of biopsy.

Imaging instrumentation. MPM was achieved using a nonlinear optical system which has been described previously (16). In brief, multiphoton images were acquired using a commercial laser scanning microscopic imaging system (Zeiss LSM 510 META; Carl Zeiss, Jena, Germany) coupled to a femtosecond Ti: sapphire laser (Mira 900-F, Coherent Inc., Santa Clara, CA, USA) operating at $800 \mathrm{~nm}$. The polarization direction of the laser light was horizontal. An oil immersion objective [x63, with numerical aperture (NA) of 1.4] was employed for focusing the excitation beam into the tissue samples (average power, $<15 \mathrm{~mW}$ ) and was also used to collect the backscattered intrinsic multiphoton signals. The images were obtained at $2.56 \mathrm{~ms}$ per pixel. A fine focusing stage (HRZ 200 stage; Carl Zeiss) was used to translate the samples following an $\mathrm{x}-\mathrm{y}$ scan of the samples to obtain a large-area image, and to change the focal position for recording various optical sections.

Histology analysis. After multiphoton imaging, histological procedures were completed including formalin fixation, paraffin embedding and 5- $\mu$ m-thick sectioning. Hematoxylin and eosin (H\&E; Dako, Carpinteria, CA, USA)-stained and antibody-stained sections were obtained from each specimen. The collagen IV primary antibody (rabbit polyclonal; 1:200 dilution; cat no. SAB4500369) was obtained from Sigma-Aldrich (St. Louis, MO, USA) and the secondary antibody (polyperoxidase rabbit anti-mouse IgG; 1:200 dilution; cat no. zs2864967) was obtained from Beijing Zhongshan
Table I. Demographic and histopathological characteristics of breast cancer patients.

\begin{tabular}{lc}
\hline Characteristic & Number of patients \\
\hline Age, years & 23 \\
$<50$ & 7 \\
$>50$ & \\
Tumor size & 12 \\
T1 & 18 \\
T2 & 0 \\
T3 & \\
Grade & 10 \\
I & 11 \\
II & 9 \\
III & \\
Tumor histology & 30 \\
IDC & \\
Estrogen receptor & 21 \\
Positive & 9 \\
Negative & \\
Progesterone receptor & 19 \\
Positive & 11 \\
Negative & \\
HER2 & \\
Positive & \\
Negative & \\
& \\
&
\end{tabular}

IDC, invasive ductal carcinoma; HER2, human epithelial growth factor receptor 2 .

Golden Bridge Biotechnology Co., Ltd. (Beijing, China). All specimens were reviewed by an attendant pathologist using a light microscope (SMZ1500; Nikon, Tokyo, Japan).

\section{Results}

Patients and tumor characteristics. The study population comprised 60 female participants who were divided into two groups. Group 1 consisted of 30 patients with breast cancer with a median age of 54 years (32-68 years). Group 2 comprised 30 patients who underwent breast reduction surgeries with a median age of 56 years (39-69 years). The average tumor size in group 1 was $2 \mathrm{~cm}$ (range, $1-5 \mathrm{~cm}$ ). The demographic and histopathological characteristics of the patients in group 1 are summarized in Table I.

Multiphoton images. To visualize the morphological features of basement membranes in normal breast and breast cancer tissue, the representative multiphoton images of the tissues are respectively shown in Fig. 2. As expected, the MPM technique visualizes the outline of basement membranes well. Large morphological differences may be observed between normal breast and breast cancer in Fig. 2. In the case of normal tissue, the normal mammary gland is a highly organized structure. The acini and ducts have a central 
A

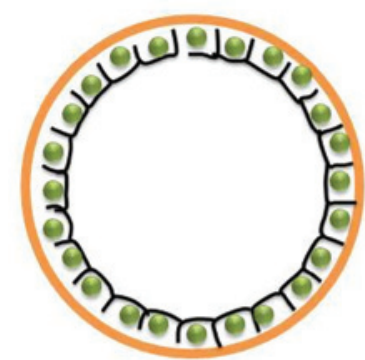

B

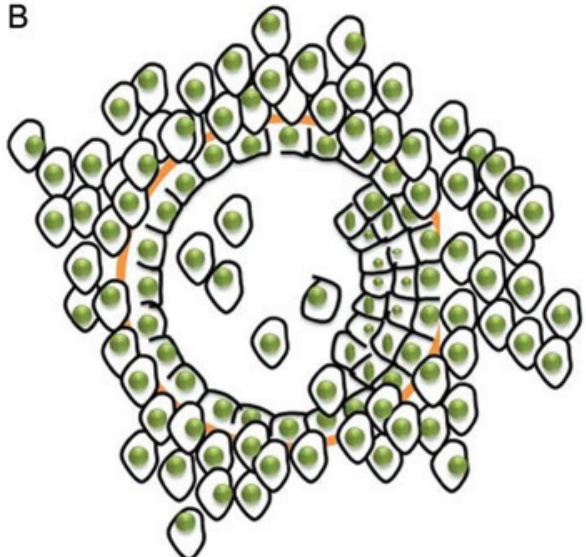

Figure 1. Schematic presentation of breast cancer progression. (A) Normal breast tissue. (B) Breast cancer tissue.
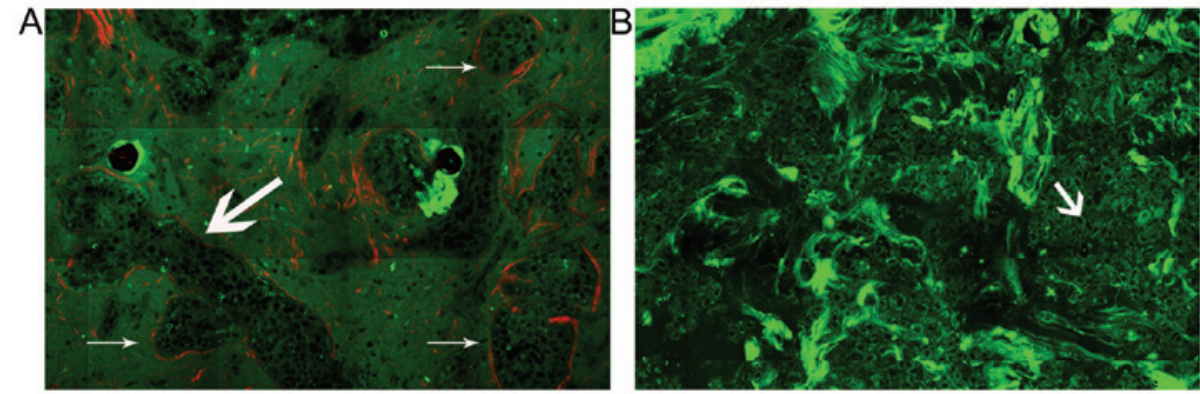

Figure 2. Representative multiphoton images from (A) normal and (B) cancerous breast tissues. To obtain a large-area image, an optional HRZ 200 fine focusing stage (HRZ 200 stage; Carl Zeiss) was used to translate the samples after the x-y scan. The excitation wavelength $\lambda_{\text {ex }}$ was 800 nm. (A) Normal breast tissue has a highly organized structure. The ducts (as shown by the large arrow) are lined by simple columnar epithelium [two-photon fluorescence (TPF) signal, in green], basement membrane (the interface of the epithelium and stroma), and stroma [second harmonic generation (SHG) signal, in red]. The basement membrane is intact and the surface is smooth and even (as indicated by the small arrow). (B) Breast carcinoma tissues lose their organized architecture. The basement membrane observed in the normal case was replaced by tumor cells (as indicated by the small arrow) and is missing. The tumor cells (TPF signal, in green) are characterized by irregular size and shape, enlarged nuclei and increased nuclear-cytoplasmic ratio. Original magnifications, x63.
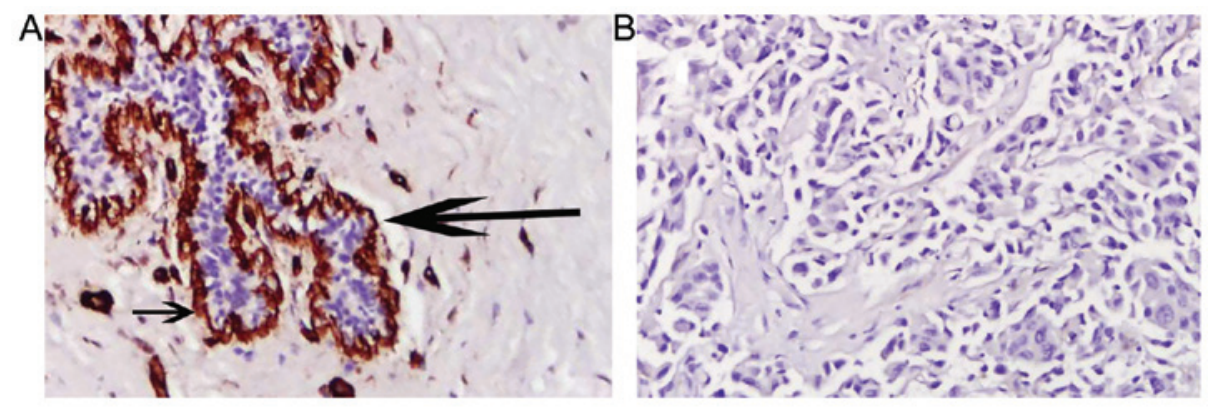

Figure 3. Typical images stained by primary antibodies to collagen IV from normal (A) and cancerous (B) breast tissues. Short arrow indicates collagen IV-positive basement membrane. Large arrow indicates ducts. Hematoxylin and eosin staining; original magnifications, $\mathrm{x} 20$.

lumen and are lined by simple columnar epithelium (TPF signal, in green), basement membrane (the interface of the epithelium and stroma), and stroma (SHG signal, in red). The basement membrane is intact and the surface is smooth and even. By contrast, breast carcinoma tissues have lost this organized architecture. The basement membrane observed in the normal case is replaced by the tumor cells and is missing. The tumor cells (TPF signal, in green) are characterized by irregular size and shape, enlarged nuclei, and increased nuclear-cytoplasmic ratio.
Histological results. The basement membrane is located between the epithelium and mesenchymal tissues. Basement membranes are considered to form a protective barrier against the initial infiltration of tissue by tumor cells. Here, immunohistochemical staining of collagen IV reveals marked differences in the organization of basement membranes in normal breast tissue and breast cancer. Continuous basement membranes with strong staining for collagen IV were observed in normal breast tissues (Fig. 3A). Conversely, breast cancer tissues were negative for collagen IV staining (Fig. 3B), which 


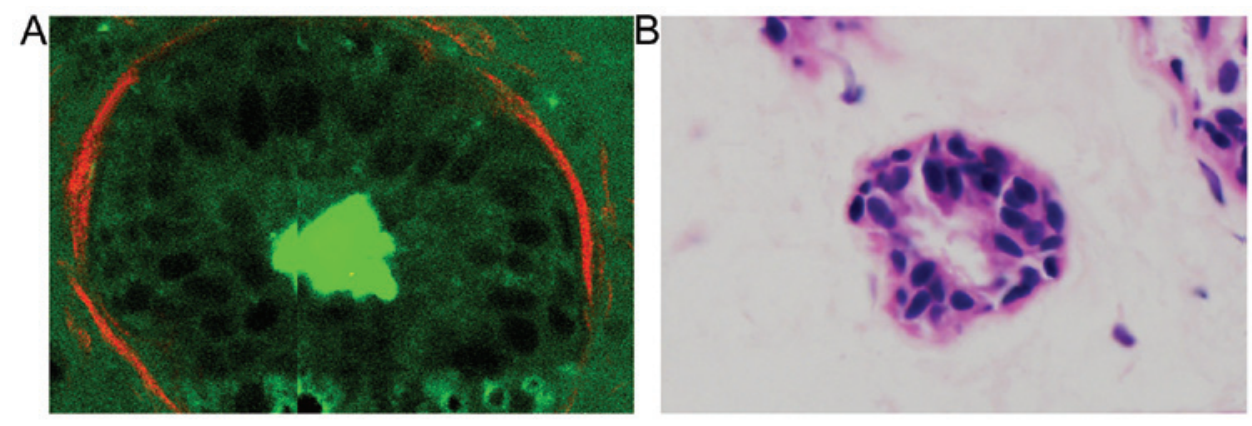

Figure 4. Comparison of multiphoton images (magnification, x63) and hematoxylin and eosin (H\&E) staining images (magnification, $\mathrm{x} 40$ ) in normal breast tissue. (A) Basement membrane (interface of epithelium and stroma) in a duct. The basement membrane is intact and smooth. (B) Corresponding H\&E image of normal breast tissue. The basement membrane is difficult to identify.

indicated the disruption and loss of basement membrane in invasive breast cancer. These results were similar to those observed with MPM imaging.

\section{Discussion}

In this study, we have provided the first evidence to demonstrate that MPM effectively characterizes basement membranes in normal (Fig. 2A) and cancerous (Fig. 2B) breast tissues. In multiphoton images, the normal breast tissue has a highly organized structure. The ducts are lined by simple columnar epithelium (TPF signal, in green), basement membrane (interface of epithelium and stroma) and stroma (SHG signal, in red). The basement membrane is intact and the surface is smooth and even (as indicated by the small arrow). By contrast, breast cancer tissues have lost this organized architecture. The basement membrane observed in the normal case is missing and replaced by tumor cells characterized by irregular size and shape, enlarged nuclei and increased nuclear-cytoplasmic ratio. These observations are in accordance with traditional histological analysis (shown in Fig. 3). The correlation between disintegration of the basement membranes of tumors and the increasingly anaplastic appearance supports the idea that basement membranes may play a role in tumor invasion $(4,17)$. Furthermore, the absence of a basement membrane barrier may facilitate tumor spread.

It was noted that MPM enables direct visualization of the basement membranes in normal and cancerous breast tissues in a manner similar to H\&E staining of conventional histological sections. Compared with $\mathrm{H} \& \mathrm{E}$ analysis, however, MPM presents a couple of advantages. Firstly, basement membrane may be clearly distinguished due to its unique structure using MPM (18), but may be difficult to identify in H\&E-stained sections (as shown in Fig. 4). Secondly, MPM introduces no artifacts during processing due to the imaging being based on intrinsic nonlinear optical contrast, making it possible to diagnose biopsy specimens in situ. Finally, the multiphoton imaging time is shorter than the turnaround time for frozen sections involving slicing, paraffin embedding or freeze-thaw processes. Therefore, MPM provides an in situ histological tool with which to evaluate basement membranes without the labeling requirement of conventional methods. The significance of this capability results from the fact that basement membranes represent a critical barrier against the initial infiltration of tissue by tumor cells $(6,7)$, and the detection of basement membrane has so far depended heavily on traditional histological procedures $(5,6)$. These results indicate that MPM holds promise for breast tissue analysis and applications to studying the dynamics of basement membrane changes at various stages of breast cancer in a manner that is compatible with clinical practice.

The use of MPM does have certain limitations in its current state. Firstly, the limited penetration depth of MPM imaging hinders evaluation of deeper tissue change. Furthermore, utilization of high NA objectives in MPM systems results in smaller fields of view, which may result in missing diseased tissues. In addition, due to sampling difficulties, this study did not include ductal carcinoma in situ, which is a proliferation of malignant epithelial cells within the mammary ductal system with intact basement membrane. The dynamic alteration of basement membrane reflects the progression of breast cancer. In future studies, we intend to focus on evaluating the feasibility of MPM for tracking the progression of breast cancer.

In conclusion, this study demonstrates the potential of MPM to visualize basement membranes, the key indicators of breast cancer progression, in a tracer-free manner. This unique method of targeting basement membrane using MPM techniques appears to be promising for further study, particularly as a complementary technique with gold-standard histopathological diagnosis. Without damaging histological procedures, this method unlocks new possibilities for in vivo diagnosis of breast cancer. With the capability of evaluating the basement membrane as shown in the present study, we envisage that a MPM-based intra-fiberoptic ductoscopy or transdermal biopsy needle (19-21) could facilitate and benefit in vivo studies and diagnoses in the years to come. Thus, in the future we intend to extend the application of MPM to in vitro breast cancer studies as well as to other types of cancer.

\section{Acknowledgements}

The present study was supported by the National Natural Science Foundation of China (81272574, 61275006 and 81271620), the Natural Science Foundation of Fujian Province (2014J01300 and 2014J05086), the Program for Changjiang Scholars and Innovative Research Team in University (IRT1115), and the Program from the Education Bureau of Fujian Province (JA12057, JA13060 and JB13127). 


\section{References}

1. Jemal A, Bray F, Center MM, Ferlay J, Ward E and Forman D: Global cancer statistics. CA Cancer J Clin 61: 69-90, 2011.

2. Eifel P, Axelson JA, Costa J, Crowley J, Curran WJ Jr, Deshler A, Fulton S, Hendricks CB, Kemeny M, Kornblith AB, et al: National institutes of health consensus development conference statement: adjuvant therapy for breast cancer, November 1-3, 2000. J Natl Cancer Inst 93: 979-989, 2001.

3. Abdelkarim M, Vintonenko N, Starzec A, Robles A, Aubert J, Martin ML, Mourah S, Podgorniak MP, Rodrigues-Ferreira S, Nahmias C, et al: Invading basement membrane matrix is sufficient for MDA-MB-231 breast cancer cells to develop a stable in vivo metastatic phenotype. PLoS One 6: e23334, 2011.

4. Plodinec M, Loparic M, Monnier CA, Obermann EC, Zanetti-Dallenbach R, Oertle P, Hyotyla JT, Aebi U, Bentires-Alj M, Lim RY and Schoenenberger CA: The nanomechanical signature of breast cancer. Nat Nanotechnol 7: 757-765, 2012.

5. Lipponen P, Ji H, Aaltomaa S and Syrjänen K: Tumour vascularity and basement membrane structure in breast cancer as related to tumour histology and prognosis. J Cancer Res Clin Oncol 120: 645-650, 1994.

6. Gusterson BA, Warburton MJ, Mitchell D, Ellison M, Neville AM and Rudland PS: Distribution of myoepithelial cells and basement membrane proteins in the normal breast and in benign and malignant breast diseases. Cancer Res 42: 4763-4770, 1982.

7. Zipfel WR, Williams RM, Christie R, Nikitin AY, Hyman BT and Webb WW: Live tissue intrinsic emission microscopy using multiphoton-excited native fluorescence and second harmonic generation. Proc Natl Acad Sci USA 100: 7075-7080, 2003.

8. Campagnola PJ and Loew LM: Second-harmonic imaging microscopy for visualizing biomolecular arrays in cells, tissues and organisms. Nat Biotechnol 21: 1356-1360,2003.

9. Zhuo S, Zheng L, Chen J, Xie S, Zhu X and Jiang X: Depth-cumulated epithelial redox ratio and stromal collagen quantity as quantitative intrinsic indicators for differentiating normal, inflammatory and dysplastic epithelial tissues. Appl Phys Lett 97: 173701, 2010.
10. Wu X, Zhuo S, Chen J and Liu N: Real-time in vivo imaging collagen in lymphedematous skin using multiphoton microscopy. Scanning 33: 463-467, 2011.

11. Hovhannisyan VA, Su PJ and Dong CY: Quantifying thermodynamics of collagen thermal denaturation by second harmonic generation imaging. Appl Phys Lett 94: 233902, 2009.

12. Matteini P,RattoF, Rossi F, Cicchi R, Stringari C, Kapsokalyvas D, Pavone FS and Pini R: Photothermally-induced disordered patterns of corneal collagen revealed by SHG imaging. Opt Express 17: 4868-4878, 2009.

13. Zhuo S, Chen J, Luo T and Zou D: Multimode nonlinear optical imaging of the dermis in ex vivo human skin based on the combination of multichannel mode and Lambda mode. Opt Express 14: 7810-7820, 2006.

14. Brown E, McKee T, diTomaso E, Pluen A, Seed B, Boucher Y and Jain RK: Dynamic imaging of collagen and its modulation in tumors in vivo using second harmonic generation. Nat Med 9: 796-800, 2003.

15. Campagnola PJ and Dong CY: Second harmonic generation microscopy: principles and applications to disease diagnosis. Laser Photonics Rev 5: 13-26, 2011.

16. Zhuo S, Chen J, Wu G, Xie S, Zheng L, Jiang X and Zhu X: Quantitatively linking collagen alteration and epithelial tumor progression by second harmonic generation microscopy. Appl Phys Lett 96: 213704, 2010.

17. Place AE, Jin Huh S and Polyak K: The microenvironment in breast cancer progression: biology and implications for treatment. Breast Cancer Res 13: 227, 2011.

18. Georgiou E, Theodossiou T and Hovhannisyan V: Second and third optical harmonic generation in type I collagen, by nanosecond laser irradiation, over a broad spectral region. Opt Commun 176: 253-260, 2000.

19. Bao H, Boussioutas A, Jeremy R, Russell S and Gu M: Second harmonic generation imaging via nonlinear endomicroscopy. Opt Express 18: 1255-1260, 2010.

20. Wu Y, Xi J, Cobb MJ and Li X: Scanning fiber-optic nonlinear endomicroscopy with miniature aspherical compound lens and multimode fiber collector. Opt Lett 34: 953-955, 2009.

21. Rivera DR, Brown CM, Ouzounov DG, Pavlova I, Kobat D, Webb WW and Xu C: Compact and flexible raster scanning multiphoton endoscope capable of imaging unstained tissue. Proc Natl Acad Sci USA 108: 17598-17603, 2011. 